\title{
Real-Time Motion Generation and Control Systems for High Wheel-Legged Robot Mobility
}

\author{
Akihiro Suzumura and Yasutaka Fujimoto, Senier Member, IEEE
}

\begin{abstract}
A wheel-legged mobile robot (WLMR) has both leg and wheel structures. WLMRs have adaptability advantages because they can change locomotion methods depending on the terrain. However, the location of a WLMR' $s$ center of gravity (CoG) is very high; Thus, almost all existing WLMRs move statically. In this study, whole body motion generation and various control systems are studied to facilitate higher WLMR mobility. To this end, a zero moment point (ZMP) is introduced as a stability index. In addition, WLMRs are modeled as single point mass linear inverted pendulums. Subsequently, online CoG pattern generation methods are proposed; one is a preview control approach and a second is an approach that realizes the desired ZMP pattern using a zero-phase low-pass filter. It is then possible to generate the desired CoG patterns more easily and faster than with a preview control approach. The CoG patterns based on the single point model are constructed via the resolved momentum control approach. Finally, the effectiveness of the whole body motion pattern generated by the proposed methods is validated by simulations and experiments.
\end{abstract}

Index Terms - Wheel-legged mobile robot, hybrid mobile robot, zero moment point, resolved momentum control, center of gravity pattern generation, zero-phase low pass filter

\section{INTRODUCTION}

$\mathbf{I}$ $\mathrm{N}$ the near future, robots will be expected to execute a variety of tasks in diverse environments. In particular, security, rescue, and life assist robots have attracted considerable attention. To meet those expectations, wheel-legged mobile robots (WLMRs) that have both leg and wheel structures have been developed [1]-[13]. There are many types of WLMR mechanisms, and almost all of them have the following favorable features: (a) stair locomotion and obstacle avoidance via a multi-degree-of-freedom leg mechanism and (b) a high energy efficiency wheel mechanism. From the above features, WLMRs have the advantages of legged and wheeled mobile robots. In addition, they have a significant mobility and adaptability advantage because they can change locomotion methods depending on the terrain. For these reasons, WLMRs have many applications including as rovers on uneven terrain and human assist robots for environmental adaptation. In our

Manuscript received October 9, 2012; revised March 16, 2013 and July 22, 2013. Accepted for publication September 4, 2013.

Copyright (C) 2013 IEEE. Personal use of this material is permitted. However, permission to use this material for any other purposes must be obtained from the IEEE by sending a request to pubs-permissions@ieee.org

A. Suzumura was with the Department of Electrical and Computer Engineering, Yokohama National University, Yokohama 240-8501 Japan. He is now with the Denso Co., Ltd., Aichi 448-8661, Japan.

Y. Fujimoto is with the Department of Electrical and Computer Engineering, Yokohama National University, Yokohama 240-8501 Japan (e-mail: fujimoto@ynu.ac.jp). study, we focus on WLMR applications for human assist purposes [14]. Adaptability is one of the most important factors in human assist robots because these robots are required to adapt to a variety of environments. In addition, speed of motion is also important. Because moving robots are at risk of colliding with humans, they require sufficient agility to avoid such collisions. Moreover, mobile robots that follow humans (e.g., luggage carrying robots) should be capable of keeping up with the humans [15]. Therefore, in addition to being adaptable, it is required that WLMRs be quick and agile.

In this study, we focus on three-dimensional (3D) WLMRs [16] [17]. The center of gravity (CoG) of such robots is inevitably quite high. Thus, there is a constant risk of falling over. Therefore, almost all 3D-WLMRs move statically and exhibit only adaptability. To obtain high mobility, the zero moment point (ZMP), which is generally used as a stability index for bipedal robots [18]-[20], is applied to the 3DWLMR. It is anticipated that the ZMP-based 3D-WLMR will move faster and more stable than the conventional 3D-WLMR.

There have been several studies that focused on increasing the speed, stability of robots such as rovers [21] and mobile manipulators [22], [23]. In addition, An et al. investigated ways of increasing the speed of WLMRs on the basis the ZMP [13]. They realized the ZMP control of a WLMR by feedforward and feedback compensation using a precise dynamical model. This approach needs the explicit formulas of the dynamical model. However, the number of degreesof-freedom (DoF) of the robot increased, complicating the situation. Furthermore, their approach was validated for only two-dimensional simulations, and no validation experiments were conducted. On the contrary, we achieved whole body motion generation and high mobility control based on the approach similar to the case of biped robots. More specifically, the WLMR is approximated as a single point mass linear inverted pendulum (LIP). Then, the CoG pattern that produces the desired ZMP pattern is generated. A preview controller is usually used to generate the CoG pattern online [24] [25]. In this study, we proposed two CoG pattern generation methods for WLMR; the first is a method based on a preview control and the other is a method based on a zero-phase low-pass filter (ZPLPF). The ZPLPF can generate the CoG pattern more easily and faster than the preview controller. The effectiveness of these proposed methods is validated based on the root mean square error (RMSE) of ZMP tracking results. Next, the generated $\mathrm{CoG}$ pattern is realized by the resolved momentum control (RMC) system [30]. The RMC approach enables a robot to control its linear momentum and angular momentum around the CoG. The linear momentum Jacobian used in 
RMC includes the CoG Jacobian [26] [27]. The robot's CoG then follows the desired $\mathrm{CoG}$ pattern. In addition, the angular momentum is another important factor used to achieve high robot mobility. There are several studies on ZMP stabilizing control using an ankle joint [28], [29]. However, unlike humanoid robots, WLMRs generally do not have ankle joints and therefore cannot stabilize the ZMP using ankle joints. Nevertheless, further enhancement of the WLMR mobility with ZMP stabilization is expected by directly manipulating the angular momentum [30]-[32]. As an example, the posture of the WLMR can be controlled using the angular momentum as a control input. The angular momentum of the robot around the $\mathrm{CoG}$ can be manipulated using RMC to yield the desired value. Then, our motion generation method was implemented and 3D simulations and experiments conducted to prove its effectiveness.

The content of this paper is organized as follows. In section II, a WLMR model is developed. In section III, we propose a CoG pattern generation method using a ZPLPF. In section $\mathrm{IV}$, a whole body motion generation scheme based on RMC is described. In section V, the overall WLMR control system is outlined. In section VI, our methods are validated via simulations and experiments. Finally, the conclusions are listed in section VII.

\section{Roвot Modeling}

This section deals with WLMR kinematic modeling. The robot is shown in Fig. 1. A CAD view of the leg is in Fig. 2 and its link parameters are listed in Table. I. The 3D robot model and coordinate systems are depicted in Fig. 3. Each coordinate frame is defined as follows.

- $\Sigma_{w}$ : Coordinates of the world

- $\Sigma_{B}$ : Coordinates of the base link

- $\Sigma_{c_{i}}$ : Coordinates of the wheel contact point.

Then,

- ${ }^{x} \mathbf{p}_{y} \in \mathbb{R}^{3}$ is the position vector of the origin of $\Sigma_{y}$ with respect to $\Sigma_{x}$.

- ${ }^{x} \boldsymbol{\omega}_{y} \in \mathbb{R}^{3}$ and ${ }^{x} \mathbf{R}_{y} \in \mathbb{R}^{3 \times 3}$ are the angular velocity and rotation matrix of $\Sigma_{y}$ with respect to $\Sigma_{x}$.

The relationship between ${ }^{x} \boldsymbol{\omega}_{y}$ and ${ }^{x} \mathbf{R}_{y}$ can be expressed as

$$
{ }^{x} \dot{\mathbf{R}}_{y}={ }^{x} \omega_{y}^{\wedge} \cdot{ }^{x} \mathbf{R}_{y}
$$

where $\wedge$ is an operator that transforms a $3 \times 1$ vector into a $3 \times 3$ skew symmetric matrix equivalent to the cross product. Operator $\vee$ is defined as the inverse of $\wedge$. In this way, the cross product can be rearranged into various forms; i.e., $\mathbf{a}(\epsilon$ $\left.\mathbb{R}^{3}\right) \times \mathbf{b}\left(\in \mathbb{R}^{3}\right)=-\mathbf{b} \times \mathbf{a}=-\mathbf{b}^{\wedge} \mathbf{a}=\mathbf{a}^{\wedge} \mathbf{b}$. Other kinematic variables are defined as follows:

- $\boldsymbol{\Phi}=\left[\begin{array}{lll}\phi & \theta & \psi\end{array}\right]^{T}$ and $\phi, \theta, \psi$ are the roll, pitch, and yaw angles expressed in terms of Euler angles.

- $\boldsymbol{\theta}_{\text {legi }}=\left[\begin{array}{llll}\theta_{1 i} & \theta_{2 i} & \cdots & \theta_{(n-1) i}\end{array}\right]^{T}$ are the vectors containing the joint angles. $n$ is the number of DoFs of the leg.

For our robot, $\boldsymbol{\theta}_{\text {legi }}=\left[\begin{array}{llll}\theta_{1 i} & \theta_{2 i} & \theta_{3 i} & \theta_{4 i}\end{array}\right]^{T} . \theta_{1 i} \sim \theta_{4 i}$ are the crotch yaw, crotch roll, knee roll, and shin yaw joint rotation angle. The wheel rotation angle is denoted as $\phi_{i}$ where $i$ represents the index of the leg and each joint angle vector is summarized as $\boldsymbol{\theta}_{i}=\left[\boldsymbol{\theta}_{\text {legi }}^{T} \phi_{i}\right]^{T} \in \mathbb{R}^{n}$.
TABLE I

LINK PARAMETERS

\begin{tabular}{|c||c|c|}
\hline & Mass $[\mathrm{kg}]$ & Length $[\mathrm{m}]$ \\
\hline \hline Base & 12.0 & $0.70(\mathrm{~W}) \times 0.35(\mathrm{D}) \times 0.40(\mathrm{H})$ \\
Crotch & 0.700 & 0.150 \\
Thigh & 3.03 & 0.350 \\
Calf & 1.52 & 0.140 \\
Shin & 1.61 & 0.280 \\
Wheel & 1.88 & 0.104 \\
\hline
\end{tabular}

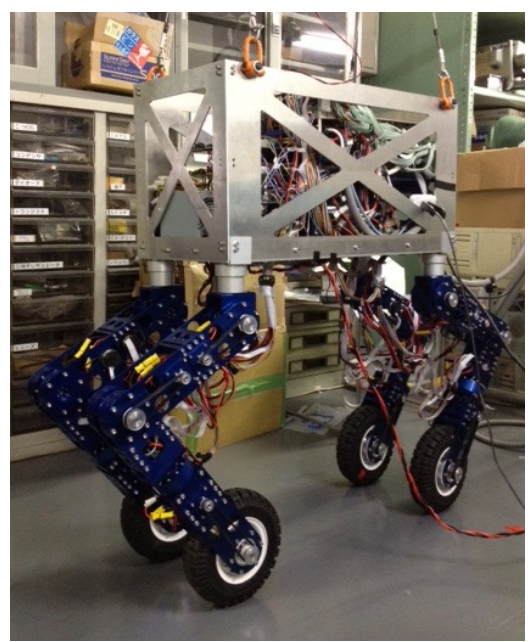

Fig. 1. An overview of the experimental setup

\section{Center of Gravity Trajectory Generation}

\section{A. Linear inverted pendulum / A Cart-table model}

In our method, a cart-table model is adopted [24]. The conceptual model is shown in Fig. 5, where we consider the $\mathrm{x}$-axis as the ordinal moving direction. In this model, the relationship between the CoG and ZMP is written as

$$
x_{z m p}=x-\frac{z_{c}}{g} \ddot{x} .
$$

where $z_{c}$ is the height of the cart's CoG, $\mathrm{g}$ is the gravitational acceleration, $\mathrm{x}$ is the $\mathrm{CoG}$ coordinate, and $x_{z m p}$ is the ZMP coordinate; Eq. (2) is called the ZMP equation.

\section{B. Preview control approach}

The preview control approach was originally proposed by Kajita et al. in [24] as a CoG trajectory generation method for humanoid robots. In this paper, we propose the use of this method for the CoG trajectory generation of WLMRs. Details of this method are described as follows.

First, Eq. (2) is translated into a state space expression as follows:

$$
\begin{aligned}
\frac{d}{d t}\left[\begin{array}{l}
x \\
\dot{x} \\
\ddot{x}
\end{array}\right] & =\left[\begin{array}{lll}
0 & 1 & 0 \\
0 & 0 & 1 \\
0 & 0 & 0
\end{array}\right]\left[\begin{array}{l}
x \\
\dot{x} \\
\ddot{x}
\end{array}\right]+\left[\begin{array}{l}
0 \\
0 \\
1
\end{array}\right] u \\
x_{z m p} & =\left[\begin{array}{lll}
1 & 0 & -\frac{z_{c}}{g}
\end{array}\right]\left[\begin{array}{c}
x \\
\dot{x} \\
\ddot{x}
\end{array}\right]
\end{aligned}
$$




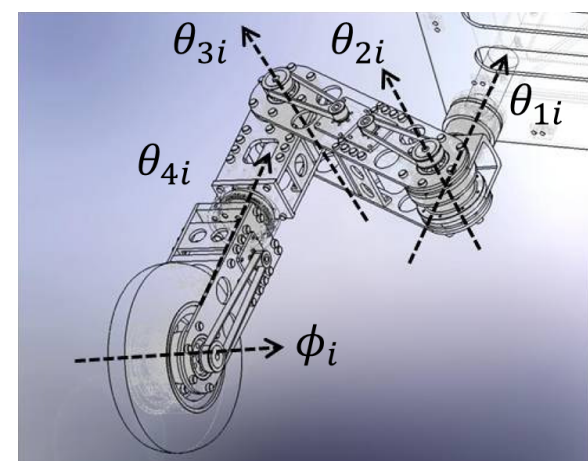

Fig. 2. CAD view of the leg mechanism

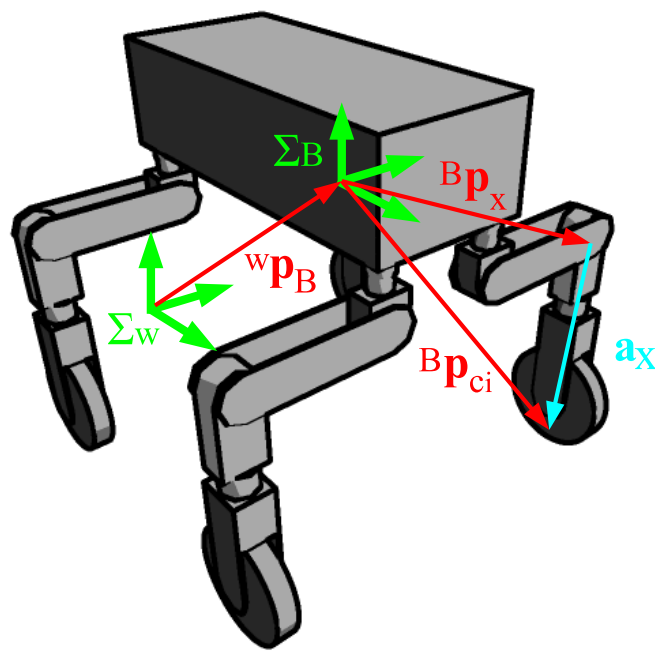

Fig. 3. Three-dimensional robot model

where control input $u$ is the time derivative of $\ddot{x}$. Eqs. (3) and (4) are then discretized with the sampling time $\Delta t$, as follows:

$$
\begin{aligned}
\mathbf{x}[k+1] & =\mathbf{A}_{d} \mathbf{x}[k]+\mathbf{B}_{d} u[k] \\
x_{z m p}[k] & =\mathbf{C}_{d} \mathbf{x}[k]
\end{aligned}
$$

where

$$
\begin{aligned}
& \mathbf{x}[k] \equiv\left[\begin{array}{lll}
x(k \Delta t) & \dot{x}[k \Delta t] & \ddot{x}(k \Delta t)^{T}
\end{array}\right] \\
& u[k] \equiv u(k \Delta t) \\
& x_{z m p}[k] \equiv x_{z m p}(k \Delta t) \\
& \mathbf{A}_{d}=\left[\begin{array}{ccc}
1 & \Delta t & \frac{\Delta t^{2}}{2} \\
0 & 1 & \Delta t \\
0 & 0 & 1
\end{array}\right], \mathbf{B}_{d}=\left[\begin{array}{c}
\frac{\Delta t^{3}}{6} \\
\frac{\Delta t^{2}}{2} \\
\Delta t
\end{array}\right] \\
& \mathbf{C}_{d}=\left[\begin{array}{lll}
1 & 0 & -\frac{z_{c}}{g}
\end{array}\right] .
\end{aligned}
$$

Next, we aim to minimize the cost function, which is written as follows:

$$
J=\sum_{k=-N_{L}+1}^{\infty}\left(Q_{e} e[k]^{2}+\Delta \mathbf{x}^{T}[k] Q_{x} \Delta \mathbf{x}[k]+R \Delta u[k]^{2}\right)
$$

where $e[k]=x_{z m p}^{r e f}[k]-x_{z m p}[k]$ is the ZMP tracking error at step $k$ and $x_{z m p}^{r e f}[k]$ is the ZMP reference. $Q_{e}, Q_{x}, R$ are the weighing coefficient for regulating each term of cost function. Then the

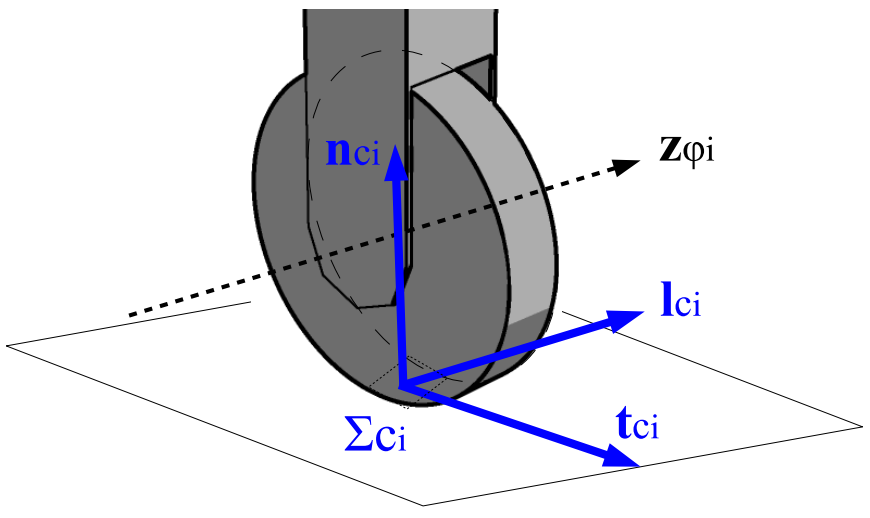

Fig. 4. Contact surface is assumed to be sufficiently stiff and flat

optimal control input $u[k]$ that minimizes the cost function is given by:

$$
u[k]=F_{e} \sum_{j=0}^{k} e[j]+F_{x} \mathbf{x}[k]+\sum_{j=1}^{M_{R}} \mathbf{F}_{R}[j] x_{z m p}^{r e f}[k+j]
$$

where $F_{e}, F_{x}$, and $\mathbf{F}_{R}[j]$ are the feedback gains and preview gains calculated from $Q_{e}, Q_{x}, R$ and $\frac{z_{c}}{g}$ based on preview control theory.

\section{Inverse system construction using zero-phase low-pass filter}

In Eq. (2), let us define the input as the $n^{\text {th }}$ differential of the CoG, $p_{C o G}^{(n)}(t)$ and the output as the $n^{\text {th }}$ differential of the ZMP, $p_{Z M P}^{(n)}(t)$. Then, the transfer function and frequency transfer function are expressed as follows:

$$
\begin{aligned}
G_{p}(s) & =\frac{s^{n} P_{Z M P}(s)}{s^{n} P_{C o G}(s)} \\
& =1-\frac{z_{c}}{g} s^{2} \\
G_{p}(j w) & =1+\frac{z_{c}}{g} \omega^{2} .
\end{aligned}
$$

where $P_{Z M P}(s)=\mathcal{L}\left[p_{Z M P}(t)\right]$ and $P_{C o G}(s)=\mathcal{L}\left[p_{C o G}(t)\right]$. We now focus on the frequency transfer function of the controlled plant. This system clearly has the characteristics of a zerophase high-pass filter (ZPHPF). The ZPHPF characteristics can be canceled by a ZPLPF used as a feedforward (FF) compensator, as described in [36]. The details of the design of the ZPLPF in the discrete time domain are explained in [37]. We define (12) as the controlled plant and the ZPLPF as the FF compensator in the discrete time domain. Then, the characteristics of the controlled plant are perfectly canceled and the ZMP pattern $p_{Z M P}^{(n)}$ follows the reference pattern. The ZMP reference $p_{Z M P}^{(n)}$ through the ZPLPF is equivalent to the desired CoG pattern $p_{C O G}^{(n)}$ because the control input is defined as the CoG pattern $p_{C o G}^{(n)}$. An outline of the ZPLPF approach is shown in Fig. 6. Later, we will show that the method can generate the desired CoG trajectory by feeding the target ZMP trajectory into the ZPLPF. Thereafter, the actual ZMP will follow the reference ZMP. We will also show that the ZPLPF can easily generate the CoG trajectory. 


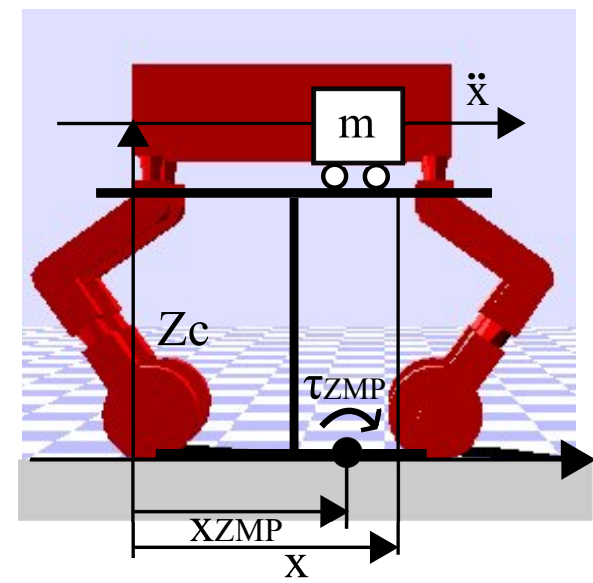

Fig. 5. Cart-table model

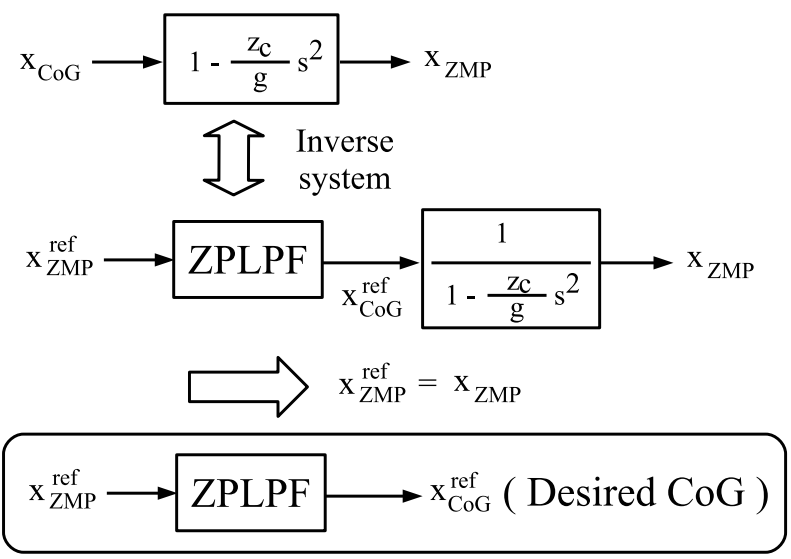

Fig. 6. Outline of the ZPLPF approach

\section{FIR-ZPLPF design}

In this subsection, the design method of the FIR-ZPLPF is described in detail. The general equation of the FIR-ZPLPF is given by

$$
G_{F I R}\left[z, z^{-1}\right]=\alpha_{0}+\sum_{k=1}^{N} \alpha_{k}\left(z^{k}+z^{-k}\right)
$$

This filter can be divided into a causal filter $G[z]$, and noncausal filter $G\left[z^{-1}\right]$, which are described as follows:

$$
\begin{aligned}
G_{F I R}\left[z, z^{-1}\right] & =a_{0}+\sum_{k=1}^{N} a_{k}\left(z^{-k}\right)+a_{0}+\sum_{k=1}^{N} a_{k}\left(z^{k}\right) \\
& =G[z]+G\left[z^{-1}\right] .
\end{aligned}
$$

where the filter coefficients of $G[z]$ and $G\left[z^{-1}\right]$ in (14) are identical. Thus, only the filter coefficients of the causal filter $G[z]$ need be designed. The noncausal filter $G\left[z^{-1}\right]$ can be realized by assuming that the input signal for the future $M^{\text {th }}$ step value is known.

Next is about the derivation of the FIR-ZPLPF coefficient. The desired filter characteristic (transfer function) is the inverse system of the controlled plant:

$$
G_{Z P L P F}(s)=\frac{1}{G_{p}(s)}=\frac{1}{1-T^{2} s^{2}} .
$$

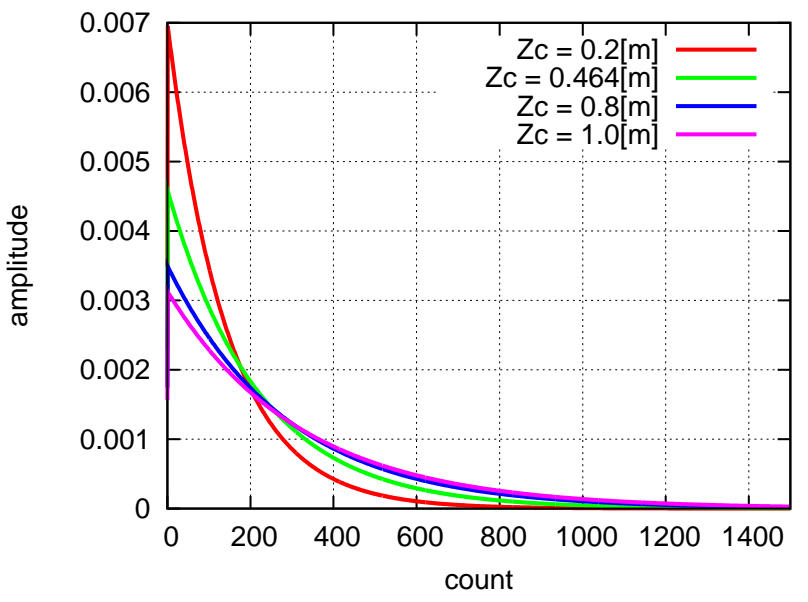

Fig. 7. Impulse response of the analog filter

where $T=\sqrt{\frac{z_{c}}{g}}$. This transfer function can be written in the form:

$$
G_{Z P L P F}(s)=\frac{1}{2}\left(\frac{1}{1+T s}+\frac{1}{1-T s}\right) .
$$

Let us now compare the above equation with (15). Then, the following transfer function is chosen as an analog prototype filter.

$$
G(s)=\frac{1}{1+T s}
$$

Thus, we obtain an FIR digital filter identical to filter (16). Next, the selected analog prototype filter $G(s)$ is discretized by the Tustin method with sampling time equal to $\Delta t$.

$$
G[z]=\frac{1+z^{-1}}{1+\frac{2 T}{\Delta t}+\left(1-\frac{2 T}{\Delta t}\right) z^{-1}} .
$$

Subsequently, we calculate the impulse response of $G[z]$ as

$$
\Lambda=\left[\begin{array}{lllll}
\delta_{0} & \delta_{1} & \delta_{2} & \ldots & \delta_{N}
\end{array}\right]
$$

where $\delta_{n}(n=0,1,2, \cdots)$ is the value at time $t=n \Delta t$. The various impulse responses of $G[z]$ are shown in Fig. 7. Next, we evaluate the equation below

$$
\tilde{a_{k}}=\sum_{k=1}^{M} \delta_{n} \delta_{n-k} .
$$

and derive the coefficient $a_{k}$ using the following equation:

$$
a_{k}=\frac{\tilde{a_{k}}}{\tilde{a_{0}}+2\left(\tilde{a_{1}}+\tilde{a_{2}}+\ldots+\tilde{a_{N}}\right)} .
$$

Following these procedures, the desired ZPLPF is calculated from (15). Further details can be found in [36] and [37]. $N$ is the number of points until the impulse response has converged.

\section{E. IIR-ZPLPF design}

Here, we describe the design method of the IIR-ZPLPF. The general equation of the IIR-ZPLPF is described as follows:

$$
G_{I I R}\left[z, z^{-1}\right]=\frac{\sum_{i=0}^{N} \beta_{i}\left(z^{i}+z^{-i}\right)}{1+\sum_{k=0}^{N} \alpha_{k}\left(z^{k}+z^{-k}\right)}
$$


This filter can be divided into a causal filter $G[z]$ and noncausal filter $G\left[z^{-1}\right]$ as follows:

$$
\begin{aligned}
G_{I I R} & =\frac{\sum_{i=0}^{N} b_{i}\left(z^{-i}\right)}{1+\sum_{k=0}^{N} a_{k}\left(z^{-k}\right)} \cdot \frac{\sum_{i=0}^{N} b_{i}\left(z^{i}\right)}{1+\sum_{k=0}^{N} a_{k}\left(z^{k}\right)} \\
& =G[z] \cdot G\left[z^{-1}\right]
\end{aligned}
$$

where the coefficients of $G[z]$ and $G\left[z^{-1}\right]$ are coincident, which is similar to the FIR-ZPLPF case. Therefore, only the design coefficients of the causal filter $G[z]$ are needed. $G\left[z^{-1}\right]$ can be composed using the first-in-last-out (FILO) method [38]. Hence, the structure of the IIR-ZPLPF can be described as shown in Fig. 8 and the characteristics of the given IIR filter are identical to (16).

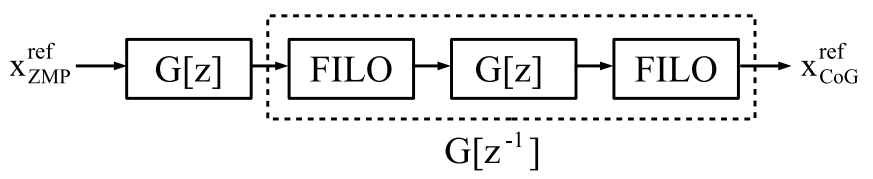

Fig. 8. Implementation of IIR-ZPLPF

Next is about the derivation of the IIR-ZPLPF coefficient. The transfer function of the desired filter is given as

$$
G_{Z P L P F}(s)=\frac{1}{1-T^{2} s^{2}}=\frac{1}{1+T s} \cdot \frac{1}{1-T s} .
$$

Let us now compare the above equation with (25). Then, the following transfer function is chosen as an analog prototype filter:

$$
G(s)=\frac{1}{1+T s}
$$

Thus, we obtain an IIR digital filter identical to filter (16). Next, the selected analog prototype filter $G(s)$ is discretized by the Tustin method. Thus, we can get $G[z]$, which is equal to (19).

\section{F. Comparison of CoG pattern generation methods}

1) Design cost: In the preview control approach, we need to solve the n-th order Riccati equation to calculate feedback gains and preview gains. In contrast to the ZPLPF approach, FIR-ZPLPF only needs to calculate the filter coefficient from the impulse response using (20), (21), and (22). The IIRZPLPF only needs to calculate the Tustin conversion of (27). Thereby, the design cost of ZPLPF is much smaller than that of the preview control approach.

2) Computational processing: The $\mathrm{CoG}$ trajectories generated by the preview control ZPLPF(FIR, IIR) are shown in Fig. 9. Here, we generate a 5 [s] CoG trajectory for the ZMP step reference under the condition that the sampling time is $\Delta t=1[\mathrm{~ms}]$ and $M=1200$. The average computation times over 100 runs for each method are 40.80 [ms], 44.12 [ms], and 0.2190 [ms] (CPU : Intel Pentium Dual Core $2.4 \mathrm{GHz}$ ). The computational processing involved in the preview control and FIR-ZPLPF are almost the same. Therefore, the advantages of the FIR-ZPLPF are the ease of theoretical development and implementation. The tap length M of the FIR-ZPLPF is large (e.g., in our case, the sum of the tap length of the causal

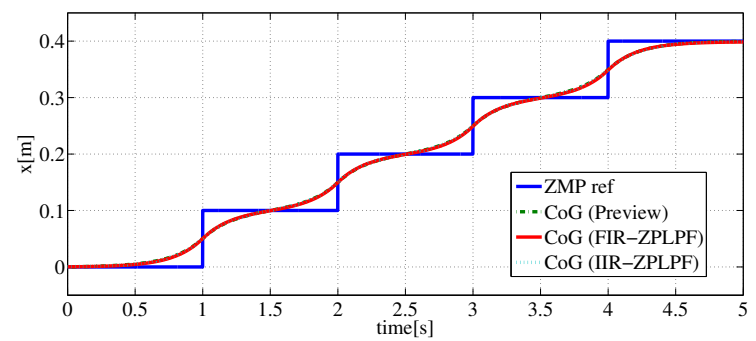

Fig. 9. Generated CoG trajectory

and noncausal filter is 2400). In contrast, the tap length of the IIR-ZPLPF is much smaller (the sum of the tap length of the causal and noncausal filter is always 2). Thus, the IIRZPLPF is superior to the conventional approach in terms of computational time. However, the IIR-ZPLPF must use the FILO method. As a result, the IIR-ZPLPF is not suitable for sequential trajectory generation at each sampling time.

\section{WHOLE-BODY MOTION GENERATION: KINEMATIC RESOLUTION}

\section{A. Non-slippage constraint for wheel-legged mechanism [8]}

In general, the kinematic constraints for WLMRs are based on the nonslippage assumption for each contact point. The slippage of contact points are described as follows:

$$
\mathbf{v}_{s_{i}}={ }^{w} \dot{\mathbf{p}}_{c_{i}}-{ }^{w} \mathbf{R}_{c_{i}}\left[\begin{array}{c}
R \dot{\phi}_{i} \\
0 \\
0
\end{array}\right]
$$

where $\mathbf{v}_{s_{i}}$ is the slippage of the $i^{\text {th }}$ leg and $\mathrm{R}$ is the wheel radius. The details of ${ }^{w} \mathbf{R}_{c_{i}}=\left[\begin{array}{ll}\mathbf{t}_{c_{i}} & \mathbf{l}_{c_{i}} \\ \mathbf{n}_{c_{i}}\end{array}\right]$ are shown in Fig. 4. Here, $\mathbf{n}_{c_{i}}$ is the contact normal vector, $\mathbf{t}_{c_{i}}$ is the longitudinal vector, and $\mathbf{l}_{c_{i}}=\mathbf{n}_{c_{i}} \times \mathbf{t}_{c_{i}}$ is the lateral vector. In this paper, we assume that the ground surface is sufficiently flat and stiff. Therefore, $\mathbf{n}_{c_{i}}$ becomes $\left[\begin{array}{lll}0 & 0 & 1\end{array}\right]^{T}$. Then, $\mathbf{t}_{c_{i}}$ is calculated as follows:

$$
\mathbf{t}_{c_{i}}=\frac{\mathbf{z}_{\phi_{i}} \times \mathbf{n}_{c_{i}}}{\left|\mathbf{z}_{\phi_{i}} \times \mathbf{n}_{c_{i}}\right|}
$$

where $\mathbf{z}_{\phi_{i}}$ is the rotational wheel axis vector. Thereafter, ${ }^{w} \dot{\mathbf{p}}_{c_{i}}$ can be described on the basis of forward kinematics as follows:

$$
\begin{aligned}
{ }^{w} \mathbf{p}_{c_{i}} & ={ }^{w} \mathbf{p}_{B}+{ }^{w} \mathbf{R}_{B}{ }^{B} \mathbf{p}_{c_{i}} \\
{ }^{w} \dot{\mathbf{p}}_{c_{i}} & ={ }^{w} \dot{\mathbf{p}}_{B}+{ }^{w} \dot{\mathbf{R}}_{B}{ }^{B} \mathbf{p}_{c_{i}}+{ }^{w} \mathbf{R}_{B}{ }^{B} \dot{\mathbf{p}}_{c_{i}} .
\end{aligned}
$$

Here, ${ }^{w} \dot{\mathbf{R}}_{B}$ can be expressed as ${ }^{w} \dot{\mathbf{R}}_{B}={ }^{w} \boldsymbol{\omega}_{B}^{\wedge} \cdot{ }^{w} \mathbf{R}_{B}$. Then,

$$
\begin{aligned}
{ }^{w} \dot{\mathbf{R}}_{B}{ }^{B} \mathbf{p}_{c_{i}} & ={ }^{w} \boldsymbol{\omega}_{B}^{\wedge} \cdot\left({ }^{w} \mathbf{R}_{B}{ }^{B} \mathbf{p}_{c_{i}}\right)=-\left({ }^{w} \mathbf{R}_{B}{ }^{B} \mathbf{p}_{c_{i}}\right){ }^{\wedge} \cdot{ }^{w} \boldsymbol{\omega}_{B} \\
& =-\left({ }^{w} \mathbf{R}_{B}{ }^{B} \mathbf{p}_{c_{i}}\right) \times{ }^{w} \boldsymbol{\omega}_{B} .
\end{aligned}
$$

${ }^{B} \dot{\mathbf{p}}_{c_{i}}$ can be described as:

$$
{ }^{B} \dot{\mathbf{p}}_{c_{i}}=\frac{\partial^{B} \mathbf{p}_{c_{i}}}{\partial \boldsymbol{\theta}_{\text {legi }}} \dot{\boldsymbol{\theta}}_{\text {legi }}=\mathbf{J}_{\text {legi } i} \dot{\boldsymbol{\theta}}_{\text {legi }} .
$$

where $\mathbf{J}_{\text {legi }} \in \mathbb{R}^{3 \times 4}$ is the Jacobian matrix, calculated as follows:

$$
\mathbf{J}_{\text {legi }}=\left[\begin{array}{llll}
\mathbf{z}_{\theta_{1 i}} \times \mathbf{a}_{\theta_{1 i}} & \mathbf{z}_{\theta_{2 i}} \times \mathbf{a}_{\theta_{2 i}} & \mathbf{z}_{\theta_{3 i}} \times \mathbf{a}_{\theta_{3 i}} & \mathbf{z}_{\theta_{4 i}} \times \mathbf{a}_{\theta_{4 i}}
\end{array}\right]
$$


where $\mathbf{z}_{x}$ is the rotation axis vector and $\mathbf{a}_{x}\left(={ }^{B} \mathbf{p}_{c_{i}}-{ }^{B} \mathbf{p}_{x}\right)$ is the vector connecting each joint center to the corresponding leg contact point. Thus, (28) can be rearranged as follows:

$$
\begin{aligned}
\mathbf{v}_{s_{i}} & ={ }^{w} \dot{\mathbf{p}}_{B}-\left({ }^{w} \mathbf{R}_{B}{ }^{B} \mathbf{p}_{c_{i}}\right) \times{ }^{w} \omega_{B} \\
& +{ }^{w} \mathbf{R}_{B} \mathbf{J}_{\text {legi }} \dot{\boldsymbol{\theta}}_{\text {legi }}-{ }^{w} \mathbf{R}_{c_{i}}\left[\begin{array}{c}
R \dot{\phi}_{i} \\
0 \\
0
\end{array}\right]
\end{aligned}
$$

In general, $\mathbf{v}_{s_{i}}$ is set to $\mathbf{0}$ to prevent slippage. Then, to simplify the calculation, the above equation is multiplied by ${ }^{w} \mathbf{R}_{B}^{T}$, yielding

$$
\begin{array}{r}
\mathbf{0}={ }^{w} \mathbf{R}_{B}^{T w} \dot{\mathbf{p}}_{B}-{ }^{B} \mathbf{p}_{c_{i}} \times{ }^{w} \omega_{B} \\
+\mathbf{J}_{\text {legi }} \dot{\boldsymbol{\theta}}_{\text {legi }}-{ }^{B} \mathbf{R}_{c_{i}}\left[\begin{array}{c}
R \dot{\phi}_{i} \\
0 \\
0
\end{array}\right]
\end{array}
$$

Then, (37) can be written in matrix form as follows:

$$
\begin{aligned}
& \mathbf{0}=\left[\begin{array}{ll}
{ }^{w} \mathbf{R}_{B}^{T} & -{ }^{B} \mathbf{p}_{c_{i}}^{\wedge}
\end{array}\right]\left[\begin{array}{c}
{ }^{w} \dot{\mathbf{p}}_{B} \\
{ }^{w} \boldsymbol{\omega}_{B}
\end{array}\right] \\
& +\left[\begin{array}{ll}
\mathbf{J}_{\text {legi }} & -{ }^{B} \mathbf{R}_{c_{i}}\left[\begin{array}{c}
R \\
0 \\
0
\end{array}\right]
\end{array}\right]\left[\begin{array}{c}
\dot{\boldsymbol{\theta}}_{\text {legi }} \\
\dot{\phi}_{i}
\end{array}\right] \\
& =\mathbf{J}_{B_{i}}^{\text {slip }} \boldsymbol{\xi}_{B}+\mathbf{J}_{\dot{\boldsymbol{\theta}}_{i}}^{\text {slip }} \dot{\boldsymbol{\theta}}_{i}=\left[\begin{array}{ll}
\mathbf{J}_{B_{i}}^{\text {slip }} & \mathbf{J}_{\dot{\boldsymbol{\theta}}_{i}}^{\text {slip }}
\end{array}\right]\left[\begin{array}{c}
\boldsymbol{\xi}_{B} \\
\dot{\boldsymbol{\theta}}_{i}
\end{array}\right]
\end{aligned}
$$

Subsequently, the leg constraint equations $(\mathrm{k}=$ total number of legs) are given by

$$
\begin{aligned}
& {\left[\begin{array}{c}
\mathbf{0} \\
\mathbf{0} \\
\vdots \\
\mathbf{0}
\end{array}\right]=\left[\begin{array}{ccccc}
\mathbf{J}_{B_{1}}^{\text {slip }} & \mathbf{J}_{\dot{\boldsymbol{\theta}}_{1}}^{\text {slip }} & \mathbf{0} & \cdots & \mathbf{0} \\
\mathbf{J}_{B_{2}}^{\text {slip }} & \mathbf{0} & \mathbf{J}_{\dot{\boldsymbol{\theta}}_{2}} \text { slip } & & \mathbf{0} \\
\vdots & \vdots & & \ddots & \\
\mathbf{\mathbf { J } _ { B _ { k } } ^ { s l i p }} & \mathbf{0} & \mathbf{0} & & \mathbf{J}_{\dot{\boldsymbol{\theta}}_{k}}^{\text {slip }}
\end{array}\right]\left[\begin{array}{c}
\boldsymbol{\xi}_{B} \\
\dot{\boldsymbol{\theta}}_{1} \\
\dot{\boldsymbol{\theta}}_{2} \\
\vdots \\
\dot{\boldsymbol{\theta}}_{k}
\end{array}\right] } \\
& \mathbf{0}=\mathbf{J}_{\text {slip }} \dot{\mathbf{q}}
\end{aligned}
$$

where, $\mathbf{J}_{B_{i}}^{\text {slip }}=\left[{ }^{w} \mathbf{R}_{B}^{T}-{ }^{B} \mathbf{p}_{c_{i}}^{\wedge}\right] \in \mathbb{R}^{3 \times 6}, \boldsymbol{\xi}_{B}=\left[{ }^{w} \dot{\mathbf{p}}_{B}^{T}{ }^{w} \boldsymbol{\omega}_{B}^{T}\right]^{T} \in \mathbb{R}^{6}$, $\mathbf{J}_{i} \in \mathbb{R}^{3 \times n}, \mathbf{J}_{\text {slip }} \in \mathbb{R}^{3 k \times(6+k n)}$ and $\mathbf{q} \in \mathbb{R}^{k n}$.

\section{B. Contact point constraint for ZMP-based wheeled locomo- tion}

In this subsection, the constraint for the contact point of each leg is defined. The objective is to constrain the ZMP at the center of the supporting polygon. To realize this, the initial posture of the robot is set to centralize the ZMP. In addition, the following constraint is imposed:

$$
\begin{aligned}
{ }^{w} \dot{\mathbf{p}}_{Z M P} & ={ }^{w} \dot{\mathbf{p}}_{c_{i}} \\
& ={ }^{w} \dot{\mathbf{p}}_{B}-\left({ }^{w} \mathbf{R}_{B}{ }^{B} \mathbf{p}_{c_{i}}\right) \times{ }^{w} \omega_{B} \\
& +\left[\begin{array}{ll}
{ }^{w} \mathbf{R}_{B}{ }^{B} \mathbf{J}_{\text {legi }} & \mathbf{0}
\end{array}\right] \dot{\boldsymbol{\theta}}_{i} \\
& =\mathbf{J}_{B_{i}}^{Z M P} \boldsymbol{\xi}_{B}+\mathbf{J}_{\dot{\boldsymbol{\theta}}_{i}}^{Z M P} \dot{\boldsymbol{\theta}}_{i}=\left[\begin{array}{ll}
\mathbf{J}_{B_{i}}^{Z M P} & \mathbf{J}_{\dot{\boldsymbol{\theta}}_{i}}^{Z M P}
\end{array}\right]\left[\begin{array}{c}
\boldsymbol{\xi}_{B} \\
\dot{\boldsymbol{\theta}}_{i}
\end{array}\right] .
\end{aligned}
$$

where $\mathbf{J}_{B_{i}}^{Z M P}=\left[\begin{array}{ll}E & -{ }^{w} \mathbf{R}_{B}{ }^{B} \mathbf{p}_{c_{i}}^{\wedge}\end{array}\right] \in \mathbb{R}^{3 \times 6}$ and $\mathbf{J}_{\dot{\boldsymbol{\theta}}_{i}}^{Z M P}=$ $\left[{ }^{w} \mathbf{R}_{B}^{B} \mathbf{J}_{\text {legi }} \mathbf{0}\right] \in \mathbb{R}^{3 \times n}$. By using this constraint, the ZMP is always set at the center of the supporting polygon. Thereafter, the leg constraint equations are described as follows:

$$
\begin{aligned}
{\left[\begin{array}{c}
{ }^{w} \dot{\mathbf{p}}_{Z M P} \\
{ }^{w} \dot{\mathbf{p}}_{Z M P} \\
\vdots \\
{ }^{w} \dot{\mathbf{p}}_{Z M P}
\end{array}\right] } & =\left[\begin{array}{ccccc}
\mathbf{J}_{B_{1}}^{Z M P} & \mathbf{J}_{\dot{\boldsymbol{\theta}}_{1}}^{Z M P} & \mathbf{0} & \cdots & \mathbf{0} \\
\mathbf{J}_{B_{2}}^{Z M P} & \mathbf{0} & \mathbf{J}_{\dot{\boldsymbol{\theta}}_{2}}^{Z M P} & & \mathbf{0} \\
\vdots & \vdots & & \ddots & \\
{ }^{w} \overline{\mathbf{p}}_{Z M P}^{Z M P} & \mathbf{0} & \mathbf{0} & & \mathbf{J}_{\dot{\boldsymbol{\theta}}_{k}}^{Z M P}
\end{array}\right]\left[\begin{array}{c}
\boldsymbol{\xi}_{B} \\
\dot{\boldsymbol{\theta}}_{1} \\
\dot{\boldsymbol{\theta}}_{2} \\
\vdots \\
\dot{\boldsymbol{\theta}}_{k}
\end{array}\right](43) \\
& =\mathbf{J}_{Z M P} \dot{\mathbf{q}}
\end{aligned}
$$

where, ${ }^{w} \overline{\mathbf{p}}_{Z M P}=\left[\begin{array}{llll}{ }^{w} \dot{\mathbf{p}}_{Z M P}^{T}{ }^{w} \dot{\mathbf{p}}_{Z M P}^{T} & \cdots & { }^{w} \dot{\mathbf{p}}_{Z M P}^{T}\end{array}\right]^{T} \in \mathbb{R}^{3 k}$ and $\mathbf{J}_{Z M P} \in \mathbb{R}^{3 k \times k n}$.

\section{Momentum Jacobian}

In general, the whole-body motion generation of a biped robot is achieved using an approximated LIP. The robot $\mathrm{CoG}$ pattern is planned on the basis of LIP. Then, the generated CoG pattern is distributed over the joint velocities by using the CoG Jacobian [26] [27]. In this subsection, the linear and angular momentum Jacobian for the 3D-WLMR are derived. The linear momentum Jacobian includes the CoG Jacobian. Therefore, the desired robot $\mathrm{CoG}$ pattern can be realized using the linear momentum Jacobian. In addition, the angular momentum around the CoG of robot and joint velocities can be mapped using the angular momentum Jacobian. Thus, the desired angular momentum can be realized using the angular momentum Jacobian.

First, the linear and angular momentum equations are derived. The linear momentum and angular momentum around the $\mathrm{CoG}$ of the robot are described as follows:

$$
\begin{aligned}
\mathbf{P} & =\sum_{i=1}^{N} m_{i}{ }^{w} \dot{\mathbf{p}}_{G_{i}} \\
\mathbf{L} & =\sum_{j=1}^{N} \mathbf{I}_{i}{ }^{w} \boldsymbol{\omega}_{i}+\left({ }^{w} \mathbf{p}_{G_{i}}-\mathbf{p}_{G}\right) \times\left(m_{i}{ }^{w} \dot{\mathbf{p}}_{G_{i}}\right)
\end{aligned}
$$

where $\mathrm{N}$ is the total number of links and ${ }^{w} \mathbf{p}_{G_{i}}$ is the $\mathrm{CoG}$ of the $i^{t h}$ link. $\mathbf{I}_{i}$ is the inertia of the $i^{\text {th }}$ link around its local origin. Then, (45) and (46) can be described in the following form:

$$
\begin{aligned}
& \mathbf{P}=\mathbf{J}_{P}(\mathbf{q}) \dot{\mathbf{q}} \\
& \mathbf{L}=\mathbf{J}_{L}(\mathbf{q}) \dot{\mathbf{q}}
\end{aligned}
$$

where $\mathbf{J}_{P}(\mathbf{q})$ and $\mathbf{J}_{L}(\mathbf{q})$ are the linear and angular momentum Jacobians. $\mathbf{J}_{P}$ and $\mathbf{J}_{L}$ can be found in various studies. Fang used the linear momentum Jacobian $\mathbf{J}_{P}$ [33] and Morita used the angular momentum Jacobian $\mathbf{J}_{L}$ [34]. Kajita et.al called $\mathbf{J}_{P}$ and $\mathbf{J}_{L}$ the "inertia matrix" [30], while Orin and Goswami called them the "Centroidal momentum matrix" [35]. In this paper, it is shown that the centroidal momentum matrix $\mathbf{A}_{G}=$ $\left[\begin{array}{ll}\mathbf{J}_{P}^{T} & \mathbf{J}_{L}^{T}\end{array}\right]^{T}$ is the product of a pure inertia matrix and a pure Jacobian matrix. The derivation of $\mathbf{J}_{P}$ and $\mathbf{J}_{L}$ are also shown in these papers. Thereby, we can obtain the joint velocities which realize the desired momentum of the WLMR using (47) and (48). 


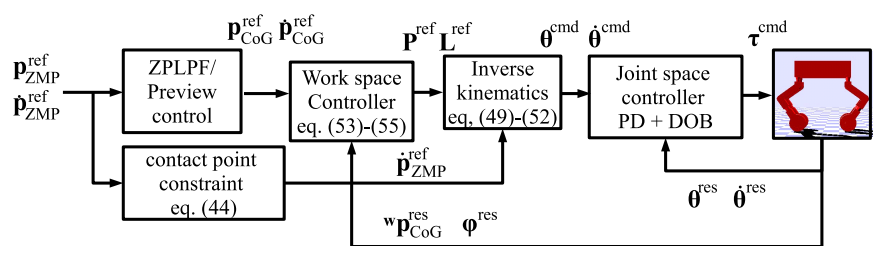

Fig. 10. Overall control block diagram

\section{Task decomposition}

In this subsection, we describe the task decomposition method and calculate the specific joint velocities that realize the desired whole body motion. The tasks defined in this paper are represented by (35), (40), (49) and (53). Here, the tasks are decomposed on the basis of the following task priorities considered in the inverse kinematics method [39]:

$$
\begin{aligned}
\dot{\mathbf{q}}^{\text {cmd }} & =\left(\mathbf{I}-\mathbf{J}_{\text {slip }}^{\dagger} \mathbf{J}_{\text {slip }}\right) \dot{\mathbf{q}}_{1} \\
\dot{\mathbf{q}}_{1} & =\mathbf{J}_{P}^{\dagger} \mathbf{P}^{\text {ref }}+\left(\mathbf{I}-\mathbf{J}_{P}^{\dagger} \mathbf{J}_{P}\right) \dot{\mathbf{q}}_{2} \\
\dot{\mathbf{q}}_{2} & =\mathbf{J}_{L}^{\dagger} \mathbf{L}^{r e f}+\left(\mathbf{I}-\mathbf{J}_{L}^{\dagger} \mathbf{J}_{L}\right) \dot{\mathbf{q}}_{3} \\
\dot{\mathbf{q}}_{3} & =\mathbf{J}_{Z M P}^{\dagger}{ }^{w} \dot{\mathbf{p}}_{Z M P}^{\text {ref }} .
\end{aligned}
$$

where $\mathbf{J}^{\dagger}$ indicates the pseudo inverse of $\mathbf{J}\left(\mathbf{J}^{\dagger}=\left(\mathbf{J}^{T} \mathbf{J}\right)^{-1} \mathbf{J}^{T}\right)$. Thus, the desired linear momentum is realized on the condition that the nonslippage constraint is achieved. In addition, the desired angular momentum and contact point constraint are realized using the remaining DoFs.

\section{Overall Control System}

To follow the position of the robot to the reference trajectory, a contact point and the $\mathrm{CoG}$ are controlled by a proportional controller described as follows:

$$
\mathbf{P}^{r e f}=m_{\text {total }}{ }^{w} \dot{\mathbf{p}}_{C o G}^{r e f}+\mathbf{K}_{p_{M}}\left({ }^{w} \mathbf{p}_{C o G}^{r e f}-{ }^{w} \mathbf{p}_{C o G}^{r e s}\right)
$$

where $m_{\text {total }}$ is the total mass of the robot and $\mathbf{K}_{p_{M}}$ is the proportional controller gain. ${ }^{w} \mathbf{p}_{C o G}^{r e f}$ and ${ }^{w} \mathbf{p}_{C O G}^{r e s}$ are the command and response positions of the $\mathrm{CoG}$ and each leg's contact point, respectively.

To control the posture of the robot to enable adaptation to uneven terrain, the reference angular momentum is generated as follows:

$$
\begin{aligned}
\mathbf{L}^{r e f} & =\mathbf{I}_{n} \boldsymbol{\omega}_{\boldsymbol{B}}^{r e f} \\
\boldsymbol{\omega}_{B}^{r e f} & =\boldsymbol{T}_{\boldsymbol{\phi}} \mathbf{K}_{p_{L}}\left(\boldsymbol{\phi}^{\text {cmd }}-\boldsymbol{\phi}^{r e s}\right)
\end{aligned}
$$

where $\boldsymbol{\phi}^{c m d}$ and $\boldsymbol{\phi}^{\text {res }}$ are the commands and responses, respectively, and $\mathbf{K}_{p_{L}}$ is the proportional gain. $\mathbf{T}_{\phi}$ is the transformation matrix between $\omega_{B}$ and $\dot{\phi}$.

With these controllers, the reference momentum around the $\mathrm{CoG}$ is calculated. Thereafter, the joint velocities are calculated by the RMC scheme and then integrated to obtain the joint angles. Finally, the joints are controlled by the proportional derivative (PD) controller and disturbance observer (DOB) [41]. The above control system is summarized in Fig. 10.

\section{Simulation and Experimental Results}

In this section, the simulation and experimental results are shown. The simulations were conducted using the 3D dynamical simulator ROCOS [42], and the experiments were conducted using the robot described in Fig. 1. The simulation and experimental condition is shown in Table. II. To verify our proposed methods, (A) posture control, (B) ZMP step response, and (C) ZMP-based wheeled locomotion tests are conducted. Their details are shown in the following subsections.

\section{A. Results of posture control}

First, the posture controller is validated by simulations and experiments. The results are shown in Figs. 11(a)-(h). Figs. 11(a)-(f) show the simulation results of the roll, pitch, and yaw angle tracking. In this simulation, the robot posture is controlled to track the desired trajectory shown in the figures. The results show that our system can control the robot posture.

Figs. 11(g) and (h) show the simulation and experimental results of the pitching control for car-like wheeled locomotion. Car-like wheeled locomotion means that the distance between the contact point of each leg and the origin of $\Sigma_{B}$ is constant. The robot $\mathrm{CoG}$ is controlled to track the reference trajectory. In this case, the reference trajectory is given by a cosine function such that the robot moves $1 \mathrm{~m}$ in the simulation and $0.6 \mathrm{~m}$ in the experiments during $1.5 \mathrm{~s}$. The results show that the robot pitch angle is suppressed by the posture controller. Thus, the effectiveness of our posture controller for wheeled locomotion is validated.

\section{B. Step response of $Z M P$}

In this subsection, we describe the simulation and experimental results of the ZMP step response. In the simulations and experiments, the control sampling time is $1[\mathrm{~ms}]$, the $\mathrm{CoG}$ height of the cart is $0.464[\mathrm{~m}]$, and $\mathrm{M}=1200$. The reference CoG trajectory generated by the preview control and ZPLPF is employed, and the CoG is controlled using the RMC scheme. Each contact point is fixed to the initial position. Here, the ZMP is calculated using the ZMP equation of a multilink model [40].

The simulation results are shown in Figs. 12(a)-(b) and the root mean square error of the ZMP tracking is shown in Table III. The results show that some overshoots are present, but that the actual ZMP follows the reference ZMP. Thus, when the CoG trajectory generated by the cart-table model approximated as a single point mass model is used, the actual ZMP follows the reference ZMP quite well.

Figs. 12(c) and (d) show the experimental results of the ZMP step response with the proposed methods. In this figure, the actual ZMP follows the reference ZMP. Thus, the CoG trajectory generated by the proposed methods is the desired trajectory. The RMSE of the ZMP tracking result is shown in Table III. Here, experiments were conducted 5 times, and an average of their RMSE is shown. The result is almost the same as that of the proposed methods. Thus, we can confirm the effectiveness of the control system based on the RMC scheme and the tracking performance of the proposed methods in the experiment. 


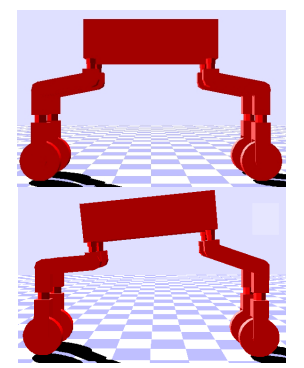

(a) Environment

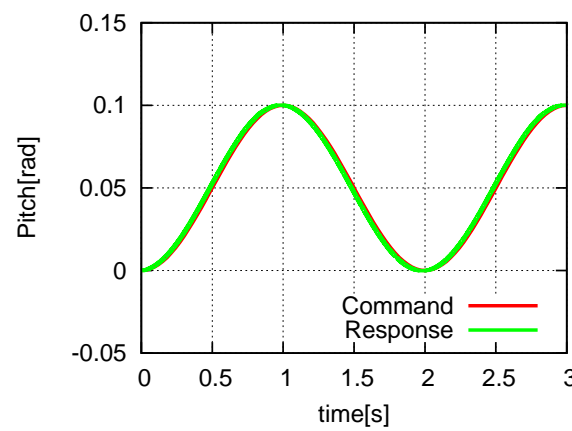

(b) Pitch angle response

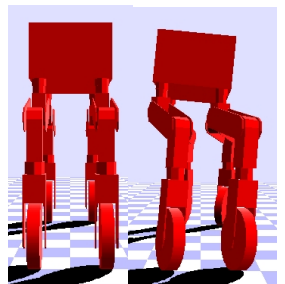

(c) Environment

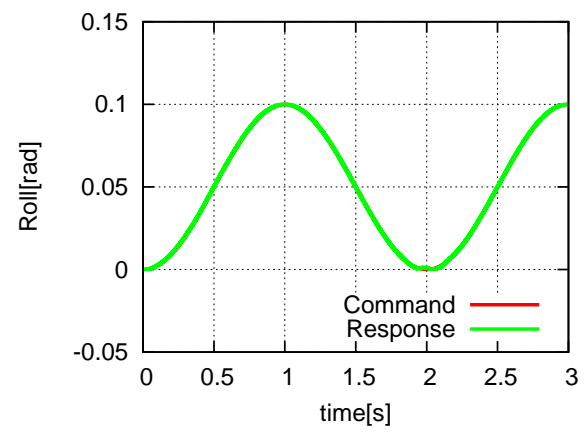

(d) Roll angle response

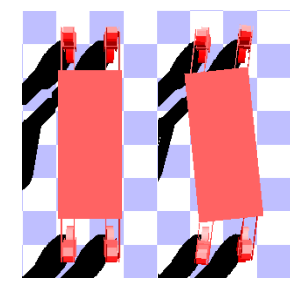

(e) Environment

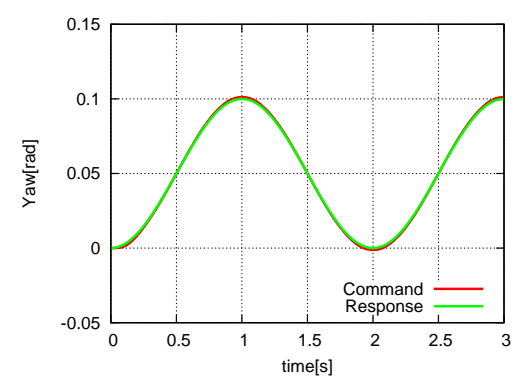

(f) Yaw angle response

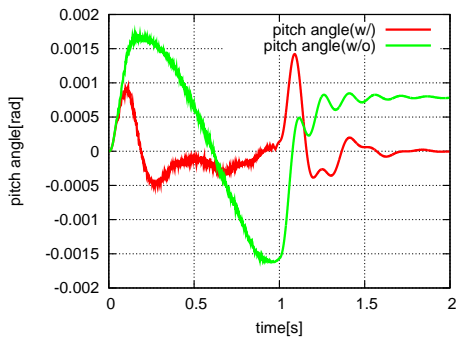

(g) Simulation : pitching comparison

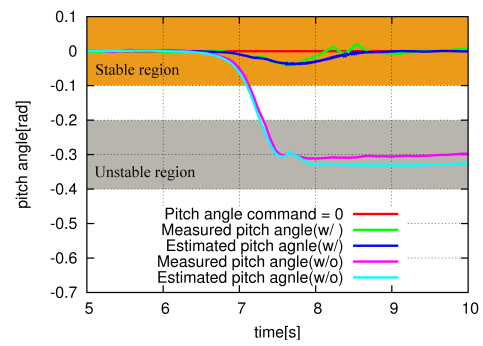

(h) Experiment : pitching comparison

Fig. 11. posture control results

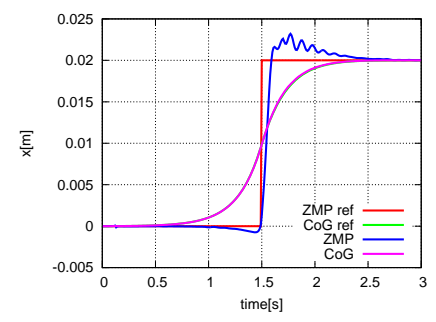

(a)Simulation

(Preview control)

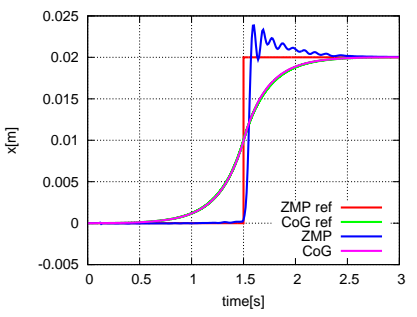

(b)Simulation (ZPLPF)

Fig. 12. Results of ZMP step response

\section{ZMP-based wheeled locomotion}

1) Without ZMP compensation: First, we show that the ZMP inverse response occurs when the robot moves like a car. The simulation and experimental results are shown in Figs. 15(a) and (b). In this case, the conditions of the simulation and experiment are the same as those for the pitching control shown in Figs. 11(g) and (h). From these results, we can confirm that the ZMP inverse response occurs and that the ZMP moves near the edge of the supporting polygon in both the simulation and experiment. In addition, the ZMP inverse response on y component of ZMP is confirmed, as shown as Figs. 16 (a) and (b). Here, the $x$ component of the ZMP is controlled to track a constant value $(0.6 \mathrm{~m} / \mathrm{s})$. The y component of the reference ZMP trajectory are given using the cosine function. The ZMP inverse response destabilizes the robot. Thus, to realize high mobility, this effect must be suppressed.

2) With ZMP compensation: Here, the result of the control for wheeled locomotion is shown. The simulation and experimental environment of wheeled locomotion is shown in Figs.
13 and 14, respectively. In this control scheme, the robot CoG is controlled to follow the reference $\mathrm{CoG}$ generated by the preview control or ZPLPF by using (53). Each contact point is constrained to centralize the ZMP inside the supporting polygon. Figs. 17(a) and 18(a) show the ZMP response of the wheeled locomotion evaluated with the proposed methods. The reference ZMP trajectory is given by a cosine function such that the robot moves $0.6 \mathrm{~m}$ during $1.25 \mathrm{~s}$. In general, the inverse response of the ZMP occurs at the high position of the CoG. However, in this case, this is prevented by our control system. From the figures, we can conclude that the proposed methods are also effective for fast-wheeled locomotion.

Figs. 17(b) and 18(b) show the experimental ZMP response. The reference ZMP trajectory is such that the robot moves $0.6 \mathrm{~m}$ during 1.25 s. Moreover, Figs. 19 and 20 show the validity of our methods for controlling the $\mathrm{y}$ component of ZMP. Here, the $\mathrm{x}$ component of ZMP are controlled to track a constant value $(0.6 \mathrm{~m} / \mathrm{s})$. The y component of the reference ZMP trajectory are given using the cosine function. These 


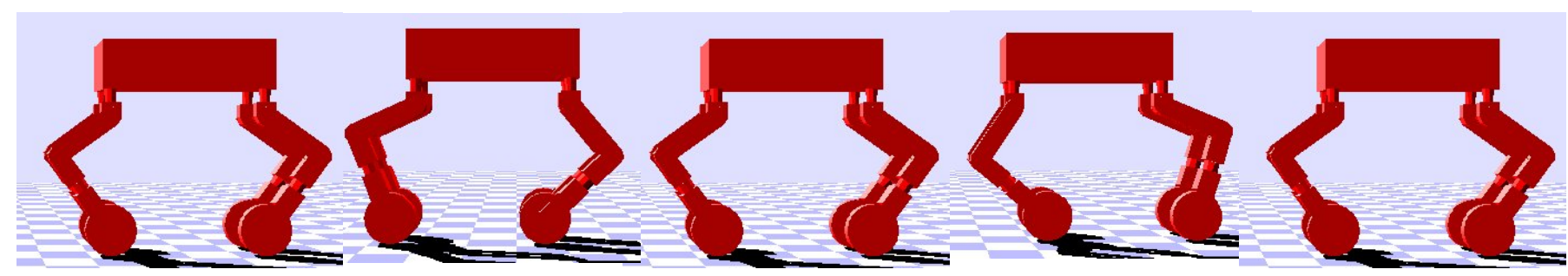

Fig. 13. Simulation environment (ZMP based wheeled locomotion)

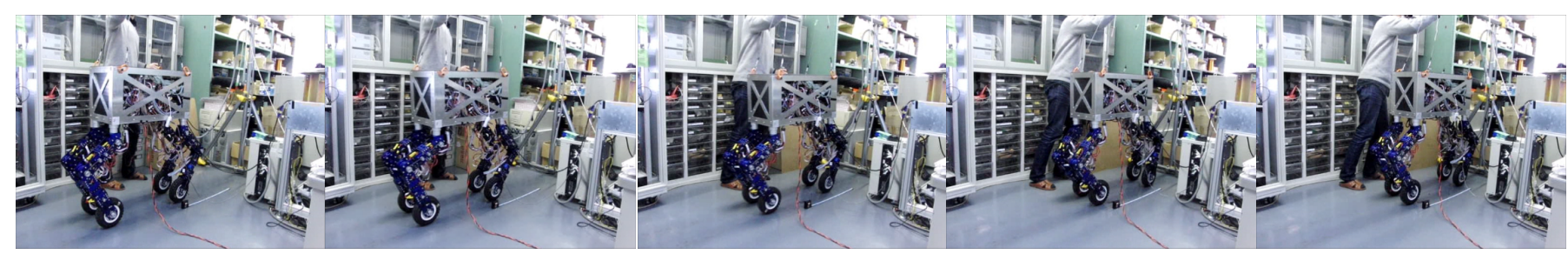

Fig. 14. Experimental environemnt (ZMP based wheeled locomotion)

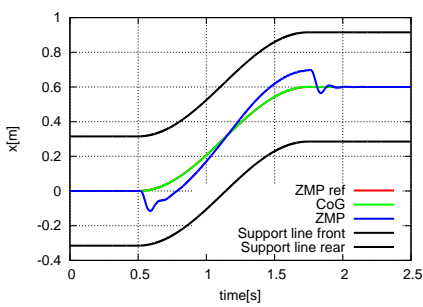

(a) Simulation result

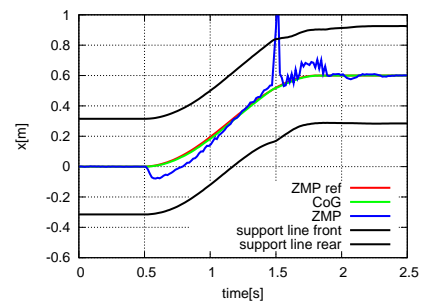

(b) Experimental result

Fig. 15. Wheeled locomotion (w/o compensation: car-like)

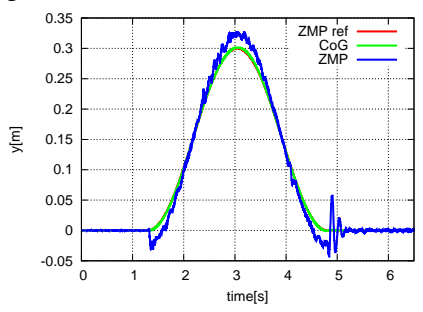

(a) Simulation result

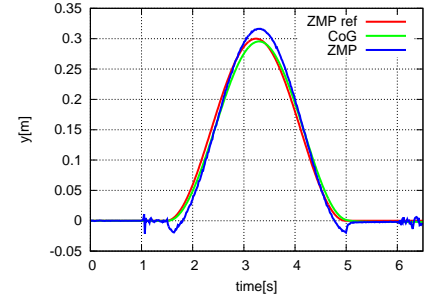

(b) Experimental result

Fig. 16. Gyrating wheeled locomotion (w/o compensation: car-like)

figures show that the ZMP response of the robot can also be experimentally suppressed by the proposed methods. The RMSE of the ZMP tracking result is shown in Table III. Here, experiments were conducted 5 times and an average of their RMSE is shown.

\section{Conclusion}

In this paper, we proposed a ZMP-based whole body motion generation and control system to produce high mobility for a 3D-WLMR. Our contributions are as follows:

- Application of inverted pendulum model for WLMR

- CoG trajectory generation based on preview control

- CoG trajectory generation based on ZPLPF (zero-phase low-pass filter)

- Whole body motion generation based on RMC (resolved momentum control)

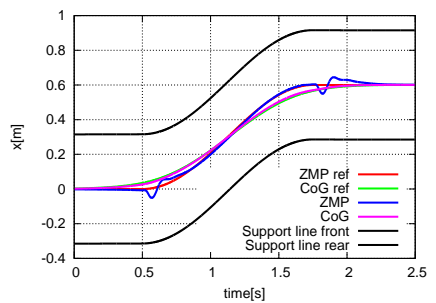

(a) Simulation result

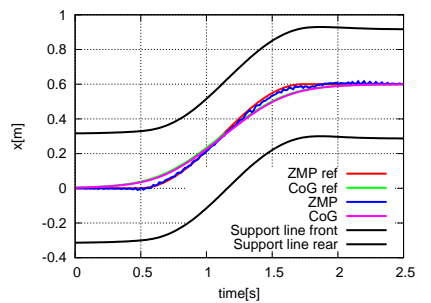

(b) Experimental result
Fig. 17. Wheeled locomotion (w/ compensation: Preview control)

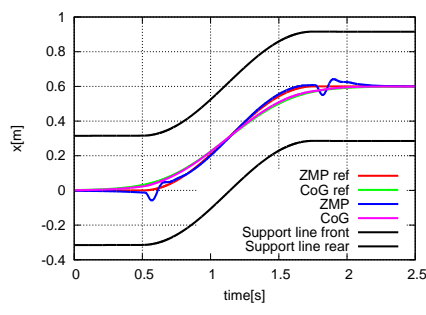

(a) Simulation result

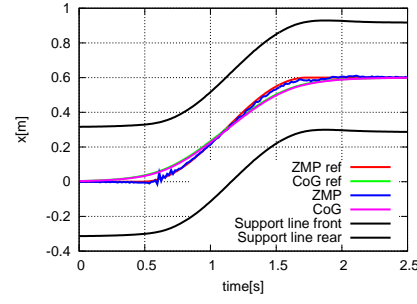

(b) Experimental result
Fig. 18. Wheeled locomotion (w/ compensation: ZPLPF)

First, the 3D-WLMR was approximated as a single point mass LIP. Then, two control approaches were proposed: the preview control approach and a new CoG pattern generation method that realizes the desired ZMP using the ZPLPF. The ZPLPF approach can generate $\mathrm{CoG}$ patterns more easily than the preview control approach. The simplicity of the theory and implementation were verified by the design methods in Section III. In addition, the computational procedures were validated by performing a CoG pattern generation test. From this test, we conclude that the IIR-ZPLPF can generate the CoG pattern faster than the preview control approach. Next, the generated CoG pattern was realized by the linear momentum Jacobian, which includes the CoG Jacobian. In addition, the angular momentum Jacobian was introduced to further increase the mobility of the WLMRs. In this paper, the posture of the WLMR is controlled using the angular momentum as a control 


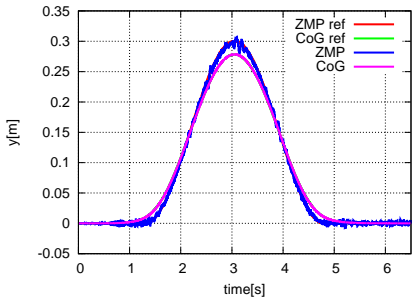

(a) Simulation result

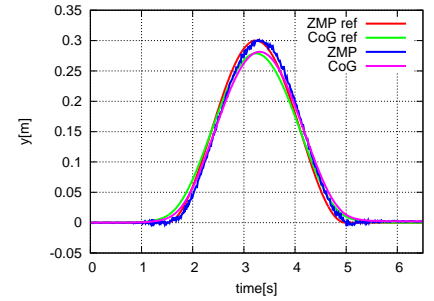

(b) Experimental result
Fig. 19. Gyrating wheeled locomotion (w/ compensation: Preview control)

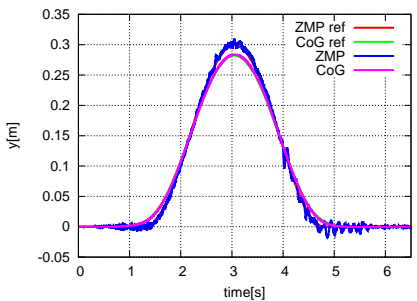

(a) Simulation result

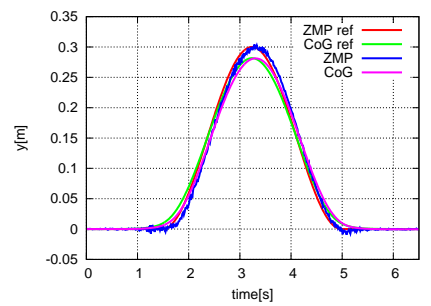

(b) Experimental result
Fig. 20. Gyrating wheeled locomotion (w/ compensation: ZPLPF)

TABLE II

Simulation and Experimental Condition

\begin{tabular}{|c||c|c|}
\hline Contents & $\begin{array}{c}\text { Simulation } \\
\text { (ROCOS) }\end{array}$ & Experiment \\
\hline \hline Sampling time[ms] $T_{s}$ & 1.000 & 1.000 \\
\hline ZPLPF tap length M & 1200 & 1200 \\
\hline Previewing period $N_{L}$ & 1200 & 1200 \\
\hline$Q_{e}$ & $1.000 \times 10^{6}$ & $1.000 \times 10^{6}$ \\
\hline$Q_{x}$ & 1.000 & 1.000 \\
\hline$R$ & 1.000 & 1.000 \\
\hline Height of CoG $z_{c}[\mathrm{~m}]$ & 0.464 & 0.464 \\
\hline Total mass $m_{\text {total }}$ & 47.0 & 47.0 \\
\hline $\mathbf{K}_{p M}$ & diag $\{10.0,10.0,10.0\}$ & diag $\{10.0,10.0,10.0\}$ \\
\hline $\mathbf{K}_{p L}$ & diag $\{10.0,10.0,10.0\}$ & diag $\{10.0,10.0,10.0\}$ \\
\hline Observer gains [rad/sec] & 100.0 & \\
-Crotch yaw & 100.0 & 100.0 \\
-Crotch roll & 100.0 & 100.0 \\
-Knee roll & 100.0 & 100.0 \\
-Shin yaw & 100.0 & 100.0 \\
-Wheel & & 100.0 \\
\hline PD gains for & & \\
joints control (Kp, Kd) & $(5.0,1.0)$ & $(5.0,3.0)$ \\
-Crotch yaw & $(350.0,50.0)$ & $(25.0,5.0)$ \\
-Crotch roll & $(200.0,80.0)$ & $(20.0,5.0)$ \\
-Knee roll & $(10.0,5.0)$ & $(50.0,5.0)$ \\
-Shin yaw & $(10.0,5.0)$ & $(15.0,5.0)$ \\
-Wheel & & \\
\hline
\end{tabular}

input. Finally, the generated CoG pattern and whole body motion generation scheme are validated by the ZMP step response and wheeled locomotion based on the ZMP. Furthermore, a ZMP-based wheeled locomotion method was proposed by manipulating the $3 \mathrm{D}-\mathrm{WLMR} \mathrm{CoG}$ and was validated. The proposed $\mathrm{CoG}$ pattern generation methods were validated via the above tests using the RMSE as an accuracy gauge. As a result, the ZMP errors of the proposed methods agree well in both simulation and experiment. Therefore, we can say that the ZPLPF approach is superior to the preview control approach in terms of implementation and computational processing.

\section{REFERENCES}

[1] B. H. Wilcox, "ATHLETE: A Cargo and Habitat Transporter for the Moon," in IEEE Aerospace Conf., pp. 1-7, 2009
TABLE III

RMSE of ZMP[Mм]

\begin{tabular}{|c||c|c|}
\hline \multicolumn{1}{|c||}{ Contents } & $\begin{array}{c}\text { Simulation } \\
\text { (ROCOS) }\end{array}$ & Experiment \\
\hline \hline ZMP step response(zplpf) & 2.445 & 2.014 \\
\hline ZMP step response(preview) & 2.217 & 1.914 \\
\hline \hline Wheeled locomotion(zplpf) & 13.71 & 9.746 \\
\hline Wheeled locomotion(preview) & 13.90 & 10.04 \\
\hline \hline Gyrat. wheeled locomotion(zplpf) & 4.886 & 10.90 \\
\hline Gyrat. wheeled locomotion(preview) & 3.516 & 10.77 \\
\hline
\end{tabular}

[2] S. Hirose, and T. Hiroki, "Study on Roller-Walk -Adaptation of Characteristics of the propulsion by a Leg Trajectory-," in Proc. IEEE ICRA, pp. 1532-1537, 2008

[3] Y. J. Dai, E. Nakano, T. Takahashi, and H. Okubo, "Motion control of legwheel robot for an unexplored outdoor environment, " in Proc. IEEE/RSJ IROS, pp. 402-409, 1998

[4] H. Adachi, N. Koyachi, T. Arai, A. Shimuzu, and Y. Nogami, "Mechanism and control of a leg-wheel hybrid mobile robot," in Proc. IEEE ICRA, pp. 1792-1797, 1999

[5] J. Smith, I. Sharf, and M. Trentini,"PAW: a Hybrid Wheeled-Leg Robot," in Proc. IEEE ICRA, pp. 4043-4048, 2006

[6] S. Moosavian, and A. Mozdbaran, "Dynamics and Motion Planning of a Wheel-Legged Mobile Robot," in Proc. IEEE CCA, pp 581-586, 2007

[7] A. Halme, I. Leppanen, S. Salmi, and S. Ylonen, "Hybrid locomotion of a wheel-legged machine," in Proc. Int. Conf. on Climbing and Walking Robots, 2000.

[8] C. Grand, F. Benamar, and F. Plumet, "Motion Kinematics Analysis of Wheeled-Legged Rover Over 3D Surface With Posture Adaptation," Int. J. Mechanism and Machine Theory, pp. 477-495, 2010

[9] T. Thomson, I. Sharf, and B. Beckman, "Kinematic Control and Posture Optimization of a Redundantly Actuated Quadruped Robot," in Proc. ICRA, pp. 1895-1900, 2012

[10] P. R. Giordano, M.Fuchs, A. Albu-Schaffer, and G. Hirzinger, "On the Kinematic Modeling and Control of a Mobile Platform Equipped with Steering Wheels and Movable Legs," in Proc. IEEE ICRA, 2009.

[11] T. Yoshioka, T. Takubo, T. Arai, and K. Inoue, "Hybrid locomotion of Leg-Wheel ASTERISK H," J. Robotics and Mechatronics, vol. 20, no. 3, pp. 403-412, 2008

[12] S. Nakajima, "RT-Mover: a rough terrain mobile robot with a simple leg-wheel hybrid mechanism," Int. Journal of Robotics Research, vol. 30, issue. 13, pp. 1609-1626, 2011

[13] S. An, Y. Oh, and D. S. Kwon, "Zero-Moment Point Based Balance Control of Leg-Wheel Hybrid Structures with Inequality Constraints of Kinodynamic Behavior," in Proc. IEEE/RSJ IROS, pp. 2471-2477, 2012.

[14] B. Jensen, N. Tomatis, L. Mayor, A. Drygajlo, and R. Siegwart, "Robot meet humans - Interaction in public spaces," IEEE Trans. on Ind. Electron., vol. 52, no. 6, pp. 1530-1546, 2005.

[15] K. Morioka, L. Joo-Ho, and H. Hashimoto, "Human-following mobile robot in a distributed intelligent sensor network," IEEE Trans. on Ind. Electron., vol.51, no.1, pp.229-237, 2004

[16] A. Suzumura, and Y. Fujimoto, "High Mobility Control for a Wheellegged Mobile Robot Based on Resolved Momentum Control," in Proc. IEEE AMC, pp. 1-6, 2012

[17] A. Suzumura, and Y. Fujimoto, "Control of Dynamic Locomotion for the Hybrid wheel-legged Robot by using Unstable-Zeros Cancellation," in Proc. IEEE ICRA, pp. 2337-2342, 2012

[18] M. Vukobratovic, and B. Borovac, "Zero-Moment Point - Thirty Five Years of its Life," Int. J. Humanoid Robot., vol. 1, no. 1, pp. 157-173, 2004.

[19] K. Erbatur, and O. Kurt, "Natural ZMP trajectories for biped robot reference generation," IEEE Trans. on Ind. Electron., vol. 56, no. 3, pp. 835-845, 2009.

[20] N. Motoi, T. Suzuki, and K. Ohnishi, "A bipedal locomotion planning based on virtual linear inverted pendulum mode," IEEE Trans. on Ind. Electron, vol. 56, no. 1, pp. 54-61, 2008.

[21] M. Krid, and F. Benamar, "Design and Control of an Active Anti-Roll System for a Fast Rover," in Proc. IEEE/RSJ IROS, pp. 274-279, 2011

[22] S. Lee, M. Leibold, M. Buss, and F. Park, "Online Stability Compensation of Mobile Manipulators Using Recursive Calculation of ZMP Gradients," in Proc. IEEE ICRA, pp. 850-855, 2012 
[23] D. Choi, and J. Oh, "ZMP stabilization of Rapid Mobile Manipulator," in Proc. IEEE ICRA, pp. 883-888, 2012

[24] S. Kajita et al., "Biped Walking Pattern Generation by using Preview Control of Zero-Moment Point," in Proc. IEEE ICRA, pp. 1620-1626, 2003

[25] S. Shimmyo, T. Sato, K. Ohnishi, "Biped Walking Pattern Generation by Using Preview Control Based on Three-Mass Model," IEEE Trans. on Ind. Electron., vol. 60, no. 11, pp. 5137-5147, 2013

[26] T. Sugihara, and Y. Nakamura, "Whole-body cooperative balancing of humanoid robot using COG Jacobian," in Proc. IEEE/RSJ IROS, vol.3, pp. 2575-2580, 2002

[27] Y. Choi, D. Kim, Y. Oh, and B. You, "Posture/Walking Control for Humanoid Robot Based on Kinematic Resolution of CoM Jacobian With Embedded Motion," IEEE Trans. Robot., Vol. 23, No. 6, pp. 1285-1293, 2007

[28] S. Kajita, M. Morisawa, K. Miura, S. Nakaoka, K. Harada, K. Kaneko, F. Kanehiro, and K. Yokoi, "Biped Walking Stabilization Based on Linear Inverted Pendulum Tracking," in Proc. IEEE/RSJ IROS, pp. 4489-4496, 2010

[29] K. Loffler, M. Gienger, F. Pfeiffer, and H. Ulbrich, "Sensors and control concept of a biped robot," IEEE Trans. on Ind. Electron., vol.51, no.5, pp. $972-980,2004$

[30] S. Kajita, F. Kanehiro, K. Kaneko, K. Fujiwara, K. Harada, K. Yokoi, and H. Hirukawa: "Resolved Momentum Control: humanoid motion planning based on the linear and angular momentum," in Proc. IEEE/RSJ IROS, vol. 2, pp. 1644-1650, 2003

[31] A. Goswami, and V. Kallem, "Rate of change of angular momentum and balance maintenance of biped robots," in Proc. IEEE ICRA, vol. 4, pp. 3785-3790, 2004

[32] A. Macchietto, V. Zordan, and C. R. Shelton, "Momentum Control for Balance," ACM Trans. Graphics, vol. 28 Issue 3, 2009

[33] A. C. Fang, and N. Pollard, "Efficient synthesis of physically valid human motion," ACM Trans. Graphics, vol.22, no. 3, pp.417-426, 2003

[34] Y. Morita, and K. Ohnishi, "Attitude control of hopping robot using angular momentum," in Proc. IEEE ICIT, vol. 1, pp. 173-178, 2003

[35] D. E. Orin, A. Goswami, and Sung-Hee Lee, "Centroidal dynamics of a humanoid robot," J. Autonomous Robots, vol. 35, issue 2-3, pp. 161-176, 2013

[36] T. Shiraishi, and H. Fujimoto, "Trajectory Tracking Control Method Based on Zero-Phase Minimum-Phase Factorization for NonminimumPhase Continuous-Time System," in Proc. IEEE AMC, pp. 1-6, 2012

[37] C. J. Kempf, and S. Kobayashi: "Disturbance Observer and Feedforward Design for a High-Speed Direct-Drive Posisioning Table," IEEE Trans. Contr. Sys. Tech., vol.7, no.5, pp.513-526, 1999

[38] L. R. Rabiner, and B. Gold, "Theory and application of Digital Signal Processing," Prentice-Hall, Inc. Englewood Cliffs, N.J.
[39] Y. Nakamura, H. Hanafusa, and T. Yoshikawa, "Task Priority Based Redundancy Control of Robot Manipulators," Int. J. Robotics Research, Vol.6, No.2, pp.3-15, 1987.

[40] A. Takanishi, M. Ishida, Y. Yamazaki, and I. Kato, "The Realization of Dynamic Walking by the Biped Walking Robot," in Proc. IEEE ICRA, pp. 459-466, 1985

[41] K. Ohnishi, M.Shibata, and T.Murakami, "Motion control for advanced mechatronics," IEEE/ASME Trans. Mechatronics, vol. 1, no. 1, pp.56-67, 1996

[42] Y. Fujimoto, and A. Kawamura, "Simulation of an Autonomous Biped Walking Robot Including Environmental Force Interaction," in IEEE Robotics and Automation Magazine, Vol. 5, No. 2, pp.33-42, 1998

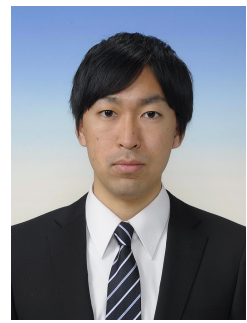

Akihiro Suzumura received the B.E. and M.E. degrees in electrical and computer engineering from Yokohama National University, Yokohama, Japan, in 2011 and 2013, respectively. Since 2013, he has been with Denso Co., Ltd., Aichi, Japan. His research interests include robotics, manufacturing automation, and motion control.

Mr. Suzumura is a member of Robotics Society of Japan.

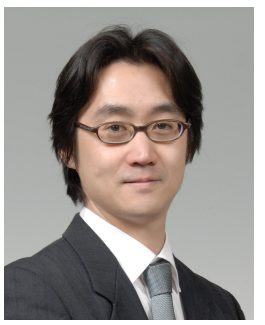

Yasutaka Fujimoto (S' 93-M ' 98) received the B.E., M.E., and Ph.D. degrees in electrical and computer engineering from Yokohama National University, Yokohama, Japan, in 1993, 1995, and 1998, respectively. In 1998, he joined the Department of Electrical Engineering, Keio University, Yokohama, Japan, as a Research Associate. Since 1999, he has been with the Department of Electrical and Computer Engineering, Yokohama National University, where he is currently a Professor. His research interests include actuators, robotics, manufacturing automation, and motion control. Dr. Fujimoto is a chair of the IEEE Industrial Electronics Society Technical Committee on Sensors and Actuators (20122013), Institute of Electrical Engineers of Japan, and the Robotics Society of Japan. 